\title{
Self-assembly of a layered two-dimensional molecularly woven fabric
}

DOI:

10.1038/s41586-020-3019-9

\section{Document Version}

Accepted author manuscript

Link to publication record in Manchester Research Explorer

\section{Citation for published version (APA):}

August, D., Dryfe, R., Haigh, S., Kent, P., Leigh, D., Lemonnier, J-F., Li, Z., Muryn, C., Palmer, L., Song, Y., Whitehead, G., \& Young, R. (2020). Self-assembly of a layered two-dimensional molecularly woven fabric. Nature: international weekly journal of science, 588(7838), 429-435. https://doi.org/10.1038/s41586-020-3019-9

\section{Published in:}

Nature: international weekly journal of science

\section{Citing this paper}

Please note that where the full-text provided on Manchester Research Explorer is the Author Accepted Manuscript or Proof version this may differ from the final Published version. If citing, it is advised that you check and use the publisher's definitive version.

\section{General rights}

Copyright and moral rights for the publications made accessible in the Research Explorer are retained by the authors and/or other copyright owners and it is a condition of accessing publications that users recognise and abide by the legal requirements associated with these rights.

\section{Takedown policy}

If you believe that this document breaches copyright please refer to the University of Manchester's Takedown Procedures [http://man.ac.uk/04Y6Bo] or contact uml.scholarlycommunications@manchester.ac.uk providing relevant details, so we can investigate your claim.

\section{OPEN ACCESS}




\section{Self-Assembly of a Layered 2D Molecularly Woven Fabric}

David P. August ${ }^{1}$, Robert A. W. Dryfe ${ }^{1}$, Sarah J. Haigh², Paige R. C. Kent ${ }^{1}$, David A. Leigh ${ }^{1 *}$, JeanFrançois Lemonnier ${ }^{1}$, Zheling $\mathrm{Li}^{2}$, Christopher A. Muryn ${ }^{1}$, Leoni I. Palmer ${ }^{1}$, Yiwei Song ${ }^{1}$, George F. S. Whitehead ${ }^{1}$ and Robert J. Young ${ }^{2}$

${ }^{1}$ Department of Chemistry, University of Manchester, Oxford Road, Manchester M13 9PL, UK. 2Department of Materials, National Graphene Institute, University of Manchester, Oxford Road, Manchester M13 9PL, UK.

*e-mail: david.leigh@manchester.ac.uk

\section{Summary Paragraph}

Fabrics, materials consisting of layers of woven fibres, are some of the most important materials in everyday life. ${ }^{1}$ Previous nanoscale weaves ${ }^{2-16}$ include isotropic crystalline covalent organic frameworks $(\mathrm{COFs})^{12-14}$ that feature rigid helical strands interlaced in all three dimensions rather than the $2 \mathrm{D}^{17,18}$ layers of flexible woven strands that give conventional textiles their characteristic flexibility, thinness, anisotropic strength and porosity. A supramolecular 2D kagome weave ${ }^{15}$ and a single-layer, surfacesupported, interwoven $2 \mathrm{D}$ polymer ${ }^{16}$ have also been reported. However, despite being proposed on a number of occasions, ${ }^{19-23}$ the direct, bottom-up, assembly of molecular building blocks into linear organic polymer chains woven in two-dimensions has remained elusive. Here we demonstrate that anion and metal ion template woven molecular 'tiles' can be tessellated into a material consisting of alternating aliphatic and aromatic segmented polymer strands, interwoven within discrete layers. Connections between slowly precipitating pre-woven grids, followed by the removal of the ion templates, results in a wholly-organic molecular material that forms as stacks and clusters of thin sheets, each sheet up to 100 s of $\mu \mathrm{m}$ long and wide but only $\sim 4 \mathrm{~nm}$ thick, in which warp and weft single-chain polymer strands remain associated through periodic mechanical entanglements within each sheet. Atomic force (AFM) and scanning electron (SEM) microscopies show clusters and, occasionally, isolated individual sheets that following demetallation have slid apart from others they were stacked with during the tessellation and polymerisation process. The layered 2D molecularly woven material has long-range order, is birefringent, twice as stiff as the constituent linear polymer, and delaminates and tears along well-defined lines in the manner of a macroscopic textile. When incorporated into a polymer-supported membrane it acts as a net, slowing the passage of large ions while letting smaller ions through. The findings open up new opportunities and research directions for molecular materials made of flexible polymer chains mechanically woven at the nanoscale in two (or three) dimensions.

Metal template synthesis is a powerful tool for directing the assembly of difficult-to-obtain molecularlevel architectures, such as the ordered entanglements necessary for molecular knots, links and other entwined structures. ${ }^{24-35}$ In principle it should be possible to tessellate pre-woven metal-coordinated molecular grids in two-dimensions to form a woven molecular material. ${ }^{20,21}$ However, to date grids featuring woven-rather than stacked ${ }^{36,37}$-ligand strands have not been reported (other than the simplest, $2 \times 2$, arrangement, which does not have internal crossings). We recently prepared a metalcoordinated interwoven $3 \times 3$ molecular grid, $\left[\mathrm{Fe}_{9} \mathbf{1}_{6}\right]\left(\mathrm{BF}_{4}\right)_{18}$, as an intermediate in the synthesis of a molecular 74 knot (Fig. 1A). ${ }^{38}$ The metal ion coordination promotes strand-crossings in the pattern required for such a knot, while the X-ray crystal structure of [Feg $\left.1_{6}\right]\left(B_{4}\right)_{18}$ (Fig. 1B-D) indicates a template effect of the $\mathrm{BF}_{4}^{-}$anions arising from the stabilisation of the orthogonal arrangement of the woven ligand strands through a combination of charge-dipole $(\mathrm{Fe}(\mathrm{II}) \cdots \mathrm{F}-\mathrm{B})$ and anion- $\pi\left(\left[\mathrm{F}_{3} \mathrm{~B}-\mathrm{F}\right]^{-}\right.$

...thiazole) interactions (Fig. 1B). The crystal packing of [Fe916] $\left(\mathrm{BF}_{4}\right)_{18}$ features layers of $\left[\mathrm{Fe}_{9} \mathbf{1}_{6}\right]^{18+}$ grids (each with four encapsulated $\mathrm{BF}_{4}^{-}$anions) with all of the ligand strands running parallel or orthogonal to the other strands in the same layer (Fig. 1C). The grid planes are separated by sheets of the remaining $\mathrm{BF}_{4}^{-}$anions (Fig. 1D). Within each grid layer the molecules are arranged in squares with the strand ends pointing across the squares towards, but slightly off-set from, the strand ends of the grid opposite (Fig. 1C). 


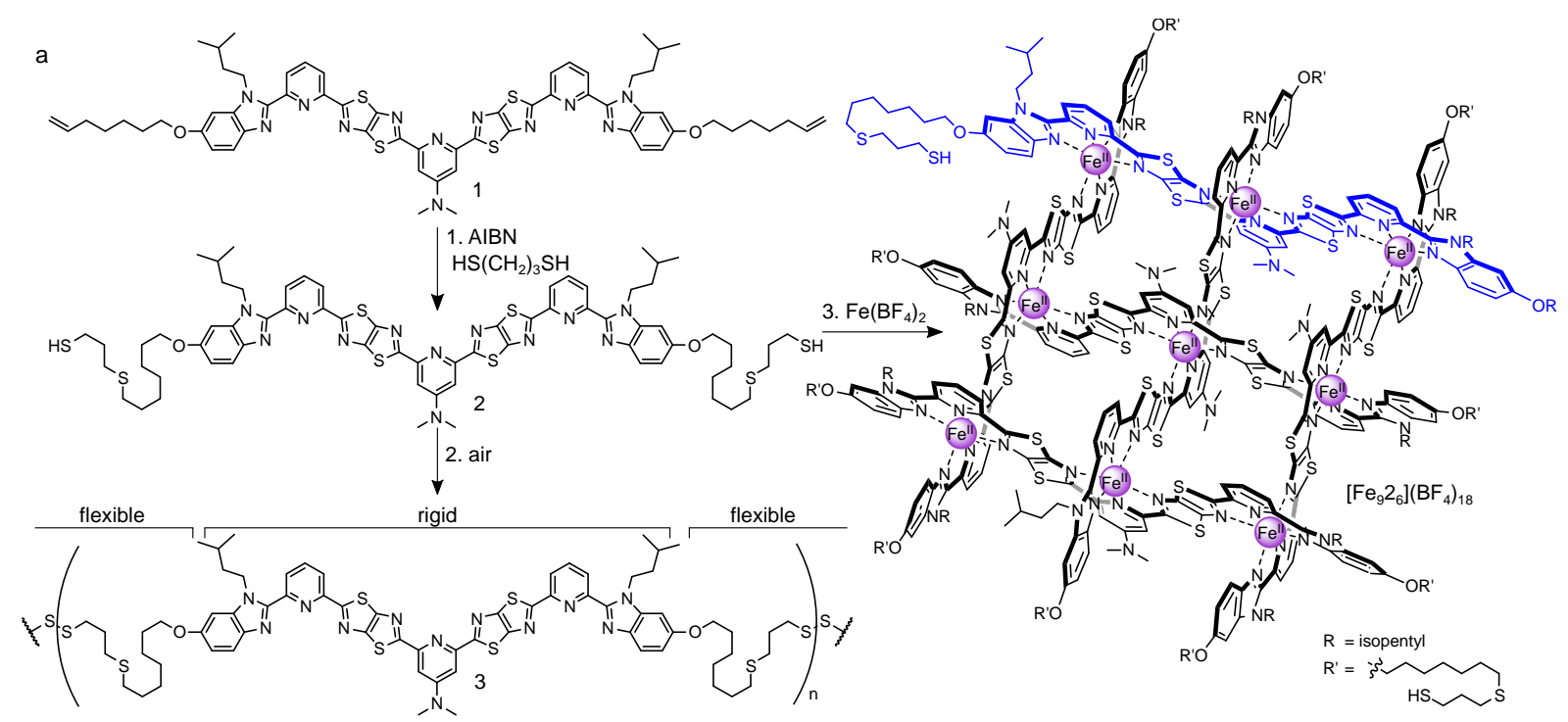

linear AABB homopolymer with alternating rigid and flexible segments
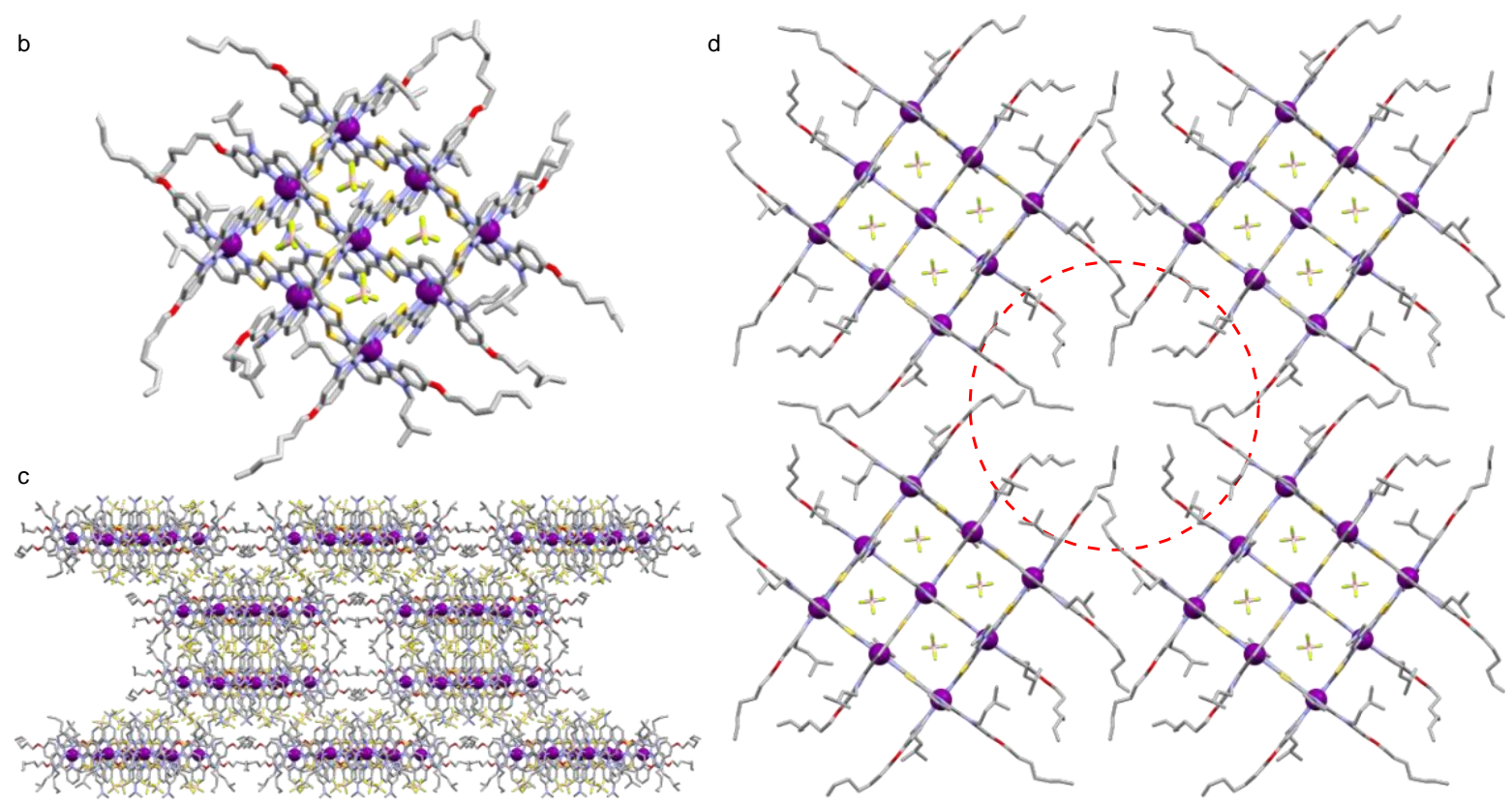

Figure 1. X-Ray crystal structure showing the layered arrangement of interwoven $3 \times 3$ molecular grid $\left[\mathrm{Fe}_{9} 1_{6}\right]\left(\mathrm{BF}_{4}\right)_{18}$, and the synthesis of thiol- and disulfide-derivatives 2, 3 and [Feg26] $\left(B_{4}\right)_{4}$ 18. (A) Synthesis of dithiol building block 2, non-woven linear AABB polymer 3, and woven $3 \times 3$ grid [Feg2 $\left.{ }_{6}\right]\left(\mathrm{BF}_{4}\right)_{18}$. Reaction conditions: (i) 1 (1.0 equiv.), $\mathrm{HS}\left(\mathrm{CH}_{2}\right)_{3} \mathrm{SH}$ (20 equiv.), 2,2'-azobis(2methylpropionitrile) (AIBN) (3 equiv.), $\mathrm{CH}_{2} \mathrm{Cl}_{2}, 80^{\circ} \mathrm{C}$ (sealed vial), 3 hours, $>98 \%$; (ii) Slow evaporation of $\mathrm{CH}_{2} \mathrm{Cl}_{2}$ solution in air; (iii) 2 (1.0 equiv.), $\mathrm{Fe}\left(\mathrm{BF}_{4}\right)_{2} \cdot 6 \mathrm{H}_{2} \mathrm{O}$ (1.5 equiv.), $\mathrm{CH}_{3} \mathrm{CN} /$ toluene $5: 3,120^{\circ} \mathrm{C}$ (sealed vial), microwave, 20 hours, $62 \%$. (B-D) X-Ray crystal structure of [Feg $\left.\mathbf{1}_{6}\right]\left(\mathrm{BF}_{4}\right)_{18 .}$. (B) View showing a single grid, [ $\left.\mathrm{Fe}_{9} \mathbf{1}_{6}\right]^{18+}$, with four encapsulated $\mathrm{BF}_{4}^{-}$anions. (C) View along the crystal $b$-axis showing layers of cationic interwoven grids separated by layers of the remaining $\mathrm{BF}_{4}$ anions. The grids crystallise in layers offset horizontally $\sim 1.8 \mathrm{~nm}$ from the closest grids in adjacent layers. Each pair of layers is offset orthogonally with respect to the next pair of layers. (D) View along the $c$-axis showing four interwoven grids and the strand end group region (red dash circle). Carbon atoms are coloured light grey; nitrogen, blue; iron, purple; oxygen, red; boron, pink; fluorine, green; sulfur, yellow. Other anions and hydrogen atoms not shown for clarity. Full experimental details are given in the Supplementary Information.

The fortuitous solid-state arrangement of $\left[\mathrm{Fe}_{9} \mathbf{1}_{6}\right]\left(\mathrm{BF}_{4}\right)_{18}$ (discrete layers, horizontally off-set layers, tiled arrangement of interwoven grids, parallel arrays of strands) suggested that connecting the strand end groups intermolecularly could result in a 2D interwoven AABB homopolymer of alternating aromatic and 
aliphatic segments, in which each weft thread crosses the warp threads by passing over one, then under the next, repeatedly, forming what is essentially a box weave (also termed a 'plain weave'). ${ }^{1}$ Indeed, perfect registry of the connections would be unnecessary to achieve a fully woven 2D material; as long as every strand end shown in the red circle in Fig. 1D is joined to another in the red circle, an extended 2D network of interwoven strands would form within each layer due to the crossings established by the $3 \times 3$ grid. After the end groups are joined and the template metal ions and anions removed, the result would be a stacked 2D molecularly woven fabric, in which the polymer strands are held together through the mechanical entanglements within each layer. Two key features of the X-ray crystal structure of $\left[\mathrm{Fe}_{9} \mathbf{1}_{6}\right]\left(\mathrm{BF}_{4}\right)_{18}$ are the planes of anions and bulky isopentyl groups between grid layers and the horizontal $\sim 1.8 \mathrm{~nm}$ off-set in grids (Fig. 1C) that alternates between the $a$ and $b$ axis for each pair of layers. With appropriate length linkers these should prevent the formation of strand connections between layers that would otherwise result in a 3D-crosslinked material.

\section{Synthesis of 2D Molecularly Woven Fabric 4 and the Corresponding Linear Polymer 3}

To promote inter-grid connections of the end groups, we used chemistry known to be effective in condensed matter, specifically the oxidation of thiols to disulfides, a reaction commonly used to crosslink proteins and other polymers. ${ }^{39} \mathrm{~A}$ useful feature of this reaction is that the only reagent, $\mathrm{O}_{2}$, and the waste product, $\mathrm{H}_{2} \mathrm{O}$, should both be able to readily diffuse through the material. Treatment of 1 with 1,3-propanedithiol $\left(\mathrm{HS}\left(\mathrm{CH}_{2}\right)_{3} \mathrm{SH}\right.$ ) and 2,2'-azobis(2-methylpropionitrile) (AIBN) in dichloromethane in a sealed vial at $80{ }^{\circ} \mathrm{C}$ afforded the dithiol building block, 2 , in near-quantitative yield through a thiolene 'click' reaction (Fig. $1 \mathrm{~A}$, step i). Treatment of 2 with $\mathrm{Fe}\left(\mathrm{BF}_{4}\right)_{2} \cdot 6 \mathrm{H}_{2} \mathrm{O}$ (1.5 equiv.) in toluene:acetonitrile $(3: 5)$ at $120^{\circ} \mathrm{C}$ in a sealed vial under microwave irradiation for $20 \mathrm{~h}$ generated [Feg26] $\left(\mathrm{BF}_{4}\right)_{18}$ in $62 \%$ yield (Fig. 1A, step iii; Supplementary Video 1). Electrospray ionisation mass spectrometry (ESI-MS) showed multiply-charged ions, $\left\{\left[\mathrm{Fe}_{92} 2_{6}\right]\left(\mathrm{BF}_{4}\right)\right\}^{(18-n)+}(n=7-11)$, corresponding to the loss of $\mathrm{BF}_{4}^{-}$counterions from the $3 \times 3$ grid (Fig. S3). The thiol-terminated grid oxidised in air: ESI$\mathrm{MS}$ and ${ }^{1} \mathrm{H}$ nuclear magnetic resonance (NMR) spectroscopy showed slow loss of the thiol protons and the formation of some intra-grid disulfide linkages in dilute solution over the course of a few days (Fig. S5); in the solid-state oxidation was complete within a few hours as evidenced by infrared (IR) and Raman spectroscopy (Figs. S17-19). To compare the effects of strand weaving, a non-woven linear polymer, 3, was prepared by the oxidation of $\mathbf{2}$ in the absence of metal salt templates (Fig. 1A, step ii). The molecular chains in both materials are the same AABB homopolymer, consisting of alternating short aromatic and aliphatic segments, shown for the non-woven linear polymer 3 in Fig. 1A.

Slow evaporation, open to the air, of a solution of freshly prepared $\left[\mathrm{Fe}_{9} \mathbf{2}_{6}\right]\left(\mathrm{BF}_{4}\right)_{18}$ in toluene:acetonitrile (3:5) led to steady formation (over 3 days) of a green film and microcrystalline particles on the surfaces of the reaction vessel originally in contact with the solution (Fig. 2C; Supplementary Video 1). Repeated washing of the combined precipitate with acetonitrile afforded 4.[Fe] as a dark-green solid. This material was insoluble in common organic solvents, similar to the behaviour of a crosslinked polymer. Raman and IR spectroscopy confirmed the absence of unreacted thiol groups in the material (the disulfide region is masked by other signals). Material 4. [Fe] was treated with $\mathrm{KCN}$ in dimethylsulfoxide (DMSO) at room temperature for $16 \mathrm{~h}$ to remove $\mathrm{Fe}$ (II) and other ions. After isolation and washing with both polar and apolar solvents, a wholly organic molecular fabric, 4, was isolated as a bright yellow fluorescent material in $72 \%$ yield from $\left[\mathrm{Fe}_{9} \mathbf{2}_{6}\right]\left(\mathrm{BF}_{4}\right)_{18}$ (Fig. 2D; Supplementary Video 1). 


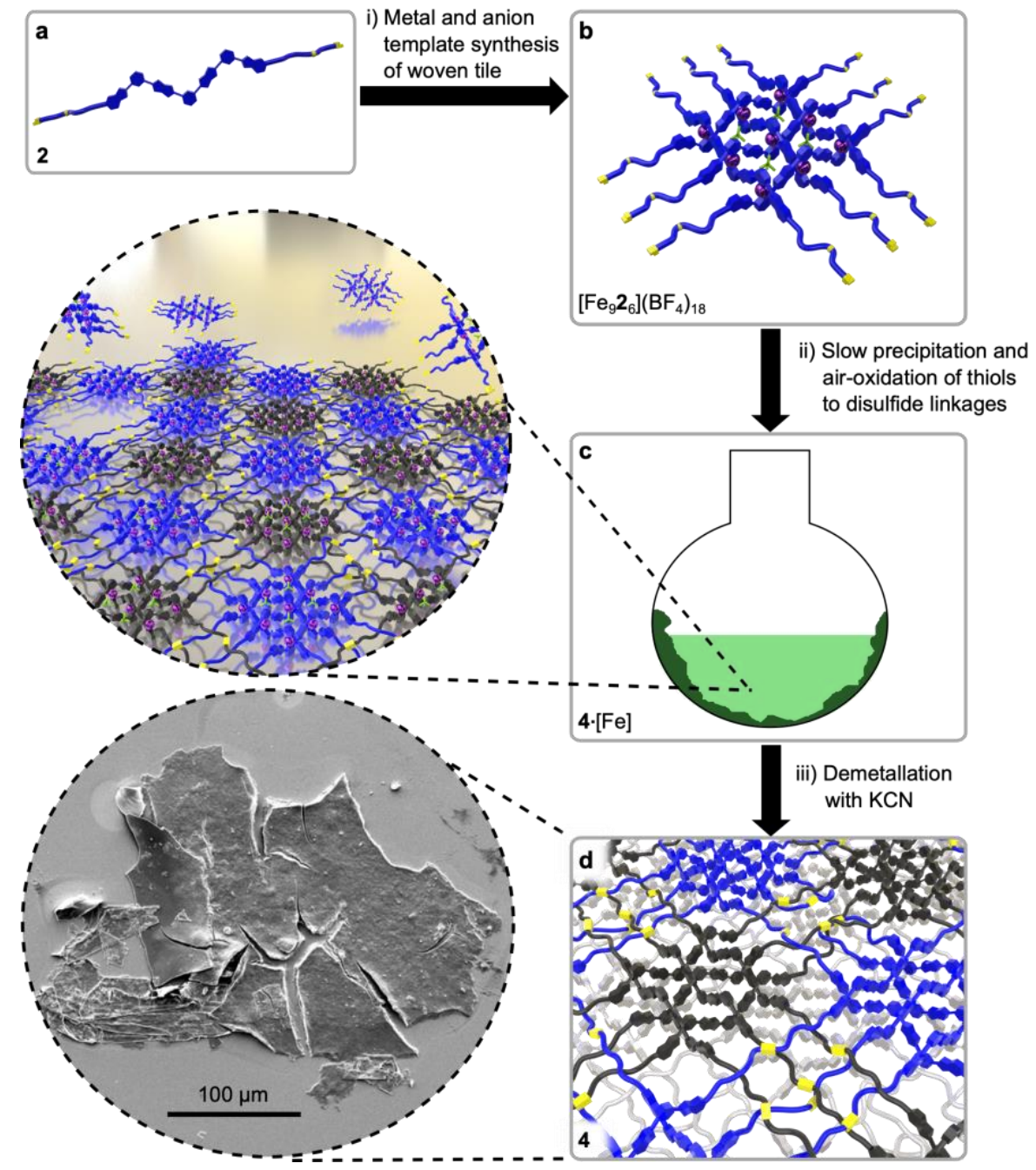

Figure 2. Bottom up, self-assembly of layered 2D molecularly woven fabric 4. (A) Strand 2, a ligand with thiol end groups. (B) Formation of $3 \times 3$ interwoven grid $\left[\mathrm{Fe}_{9} \mathbf{2}_{6}\right]\left(\mathrm{BF}_{4}\right)_{18}-\mathrm{a}$ 'woven tile'-by anion and metal template weaving of 2. (C) Slow precipitation of $\left[\mathrm{Fe}_{9} \mathbf{2}_{6}\right]\left(\mathrm{BF}_{4}\right)_{18}$ and air-oxidation of the thiol groups to disulfide bonds. Inset depicts assembly of the already-woven tiles into 2D layers, 4.[Fe]. Grids are coloured blue and grey to clarify the weaving pattern. As oxidation in solution is slow, most of the disulfide linkages probably form in the solid state. (D) Treatment of 4.[Fe] with excess $\mathrm{KCN}$ removes the template salts to afford a wholly organic molecularly woven fabric, 4 , as stacked sheets and clusters, with individual sheets having typical dimensions up to several hundreds of $\mu \mathrm{m}$ in length and width but only a few $\mathrm{nm}$ thick. Delamination (sliding of one sheet over another, e.g. Fig. 3D) indicates that stacked sheets are not vertically joined together. The strand registry is likely to have some defects within a layer (e.g. additional strand crossings, corner joins that transition strand orientation from warp-to-weft, etc, see Supplementary Information Section 1.19) beyond the nine crossings per grid set up by metal ion coordination. Extrapolating from the parent grid structure (Fig. 1), the thread count (strand density in two-dimensions, corresponding to the sum of the number of threads both lengthwise and widthwise per inch) is 40-60 million. Inset showing SEM image of 4. Experimental procedures and characterisation data are provided in the Supplementary Information. For an animation of the assembly process, see Supplementary Video 1.

\section{Structural Characterisation of 4}

The colour and fluorescence of 4 (Fig. 3A) are indicative of the absence of $\mathrm{Fe}(\mathrm{II})$ ions. Elemental analysis was in good agreement with the element stoichiometry required for $\mathbf{4}$, and energy dispersive $X$-ray spectroscopy (EDS) of $\mathbf{4}$ confirmed the effectiveness of the demetallation process with baseline values of $\mathrm{Fe} \mathrm{L}_{\alpha}$ and $\mathrm{K}_{\alpha / \beta}$ emission (Fig. 3B). 

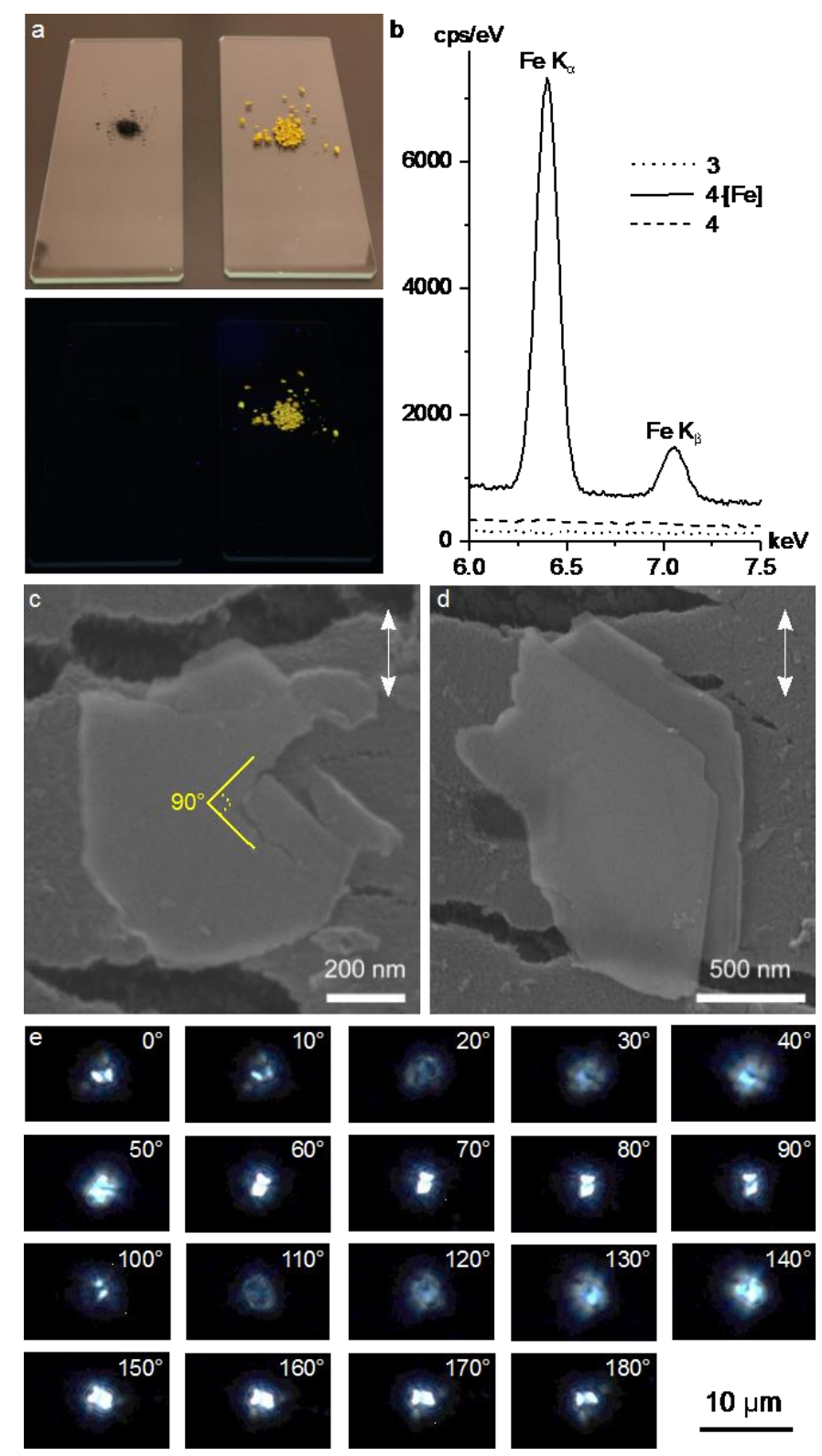

$10 \mu \mathrm{m}$

Figure 3. Images of layered 2D molecularly woven fabric 4, and evidence for long range order. (A) Top: Images of the 2D molecularly woven polymer, with metal salt still incorporated (4.[Fe], left) and demetallated and ion-free (4, right). Bottom: The same samples in the dark under UV-irradiation, showing fluorescence of the metal-free material, 4. (B) Energy dispersive X-ray spectroscopy (EDS) emission line data at $\mathrm{K}_{\alpha}(6.40 \mathrm{keV})$ and $\mathrm{K}_{\beta}(7.06 \mathrm{keV})$ for $4 \cdot[\mathrm{Fe}]$ and the metal-free materials, unwoven linear polymer $\mathbf{3}$ and 2D molecularly woven polymer 4. (C-E) Evidence for long-range ordering in 4. (C) SEM image showing well-defined fracture lines caused by putting polymer-supported flakes of 4 under strain. White arrows indicate direction of strain. (D) SEM image showing exfoliation of whole layers caused by putting polymer-supported flakes of $\mathbf{4}$ under strain. White arrows indicate direction of strain. (E) Optical images of a rotating single flake of 4 taken through polarised optical filters showing birefringence. The amount of polarised light that passes through the $2 \mathrm{D}$ molecularly woven material is similar every 90 degrees of rotation of the sample (compare images at $20^{\circ}$ and $110^{\circ}$ (dark) and $60^{\circ}$ or $70^{\circ}$ and $150^{\circ}$ or $160^{\circ}$ (bright)), indicative of aligned polymer chains. For an animation of the fracturing and delamination processes that result in images (C) and (D), see Supplementary Video 3.

Polymers consisting of alternating short aromatic-aliphatic segments rarely form flake-like morphologies, but the demetallated material, $\mathbf{4}$, was isolated as large, very thin, flakes and free-standing nanosheets that formed spontaneously without the need for standard exfoliation methods such as sonication or manual grinding. ${ }^{18}$ The nanosheets were separated from larger flakes by suspending in 
methanol and centrifugation at $1500 \mathrm{rpm}$. The supernatant liquid displayed the characteristic Tyndall scattering associated with suspended nanosheets (Fig. S23).$^{40}$ AFM of samples drop-cast from a methanol suspension onto silicon wafers showed layers of nanosheets with a minimum step height of $\sim 4 \mathrm{~nm}$ (Fig. 4C and D; Supplementary Video 2). Note that the horizontal axis of the AFM trace is in $\mu \mathrm{m}$, whereas the vertical axis is in $\mathrm{nm}$ : the sheets extend for thousands of grid lengths and woven overunder crossings without a change in height. Such extended flat surfaces cannot arise from polymerisation in three-dimensions, although we do not rule out the formation of some disulfide linkages between layers within a sheet. The scarcity of divots in the AFM traces suggests that within a layer either the absence of individual tiles is rare or following demetallation the woven strands are able to move to cover small gaps through reptation (slithering movements of regions of the polymer chains within the weave) ${ }^{41}$ Sometimes wrinkles were observed in the molecular fabric as an internal region detaches and rises up from the substrate (Fig. 4C), similar to features previously observed ${ }^{42}$ with 2D graphene sheets. As a result of the process used to promote tessellation most of the sheets are present in stacks or clusters (Fig. S25A), but occasionally an isolated 4-nm-thick sheet could be imaged directly on the surface (Fig. S25B).

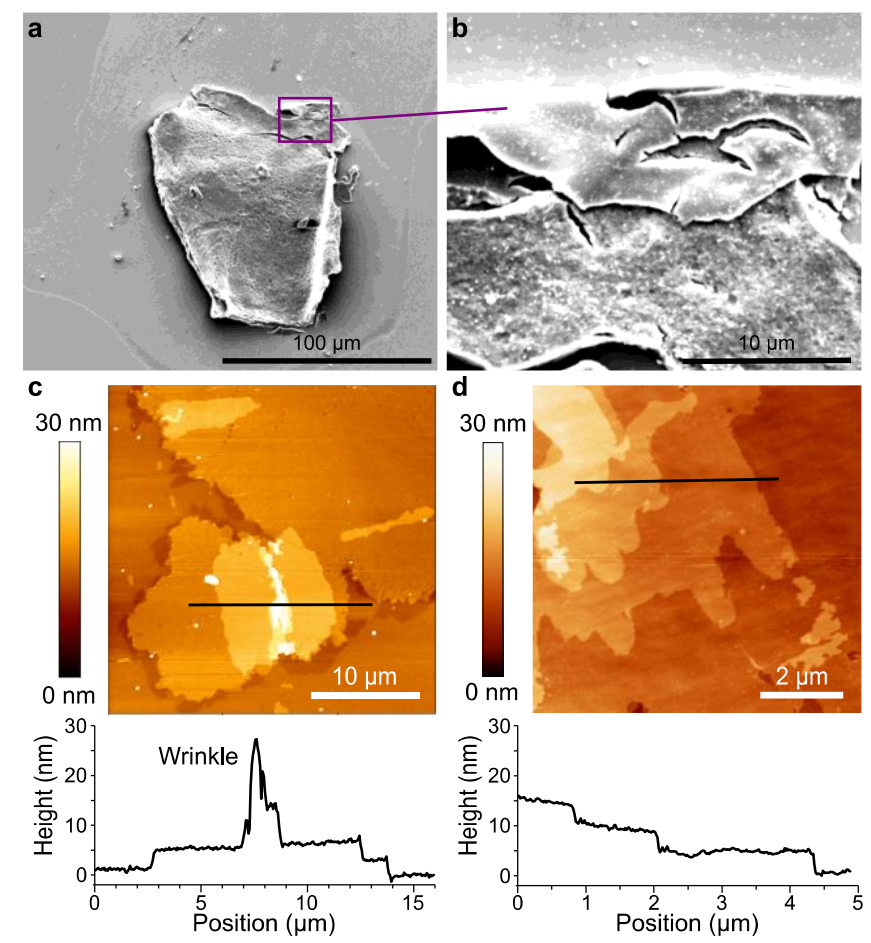

Figure 4. Microscopy imaging of layered 2D molecularly woven fabric 4. (A) SEM image of an individual large flake. (B) SEM image of a region at higher magnification, showing the thin layered structure. (C and D) AFM images of layered nanosheets of 4 dropcast onto $\mathrm{Si} / \mathrm{SiO}_{2}$ and the corresponding line-profiles showing consistent $4 \mathrm{~nm}$ step-heights. The regular height of the surface indicates that the interwoven $2 \mathrm{D}$ material generally lies flat and extended, but image $\mathbf{C}$ shows a height change corresponding to a wrinkle in the woven molecular fabric. The SEM and AFM images show the flat surface of the material extending in two dimensions for $10 \mathrm{~s}$ of $\mu \mathrm{m}$ in the 2D sheets, corresponding to tens of thousands of over-under crossings for each woven strand. For an animation of the AFM imaging a flat layered sheet, see Supplementary Video 2.

The thin flat nanosheets were also observed by SEM (Fig. 4A \& B) and optical microscopy (Fig. S24) and, again, suggest well-ordered domains brought about by effective tiling of the parent grids. Shortrange order refers to the regular arrangement of atoms over a short distance, usually one or two atom spacings, such as within the unit cell of crystals. Regularity repeated over greater distances, for example parallel arrangements of polymer chains or mesogens in liquid crystals, is termed long-range order. ${ }^{43}$ Long-range orientational order of polymer chains, often brought about in films and fibers by mechanical processing, can be demonstrated by birefringence. ${ }^{43}$ The flakes of $\mathbf{4}$ exhibit strong birefringence with maxima every $90^{\circ}$ of rotation, confirming long-range order and domains of aligned polymer strands (Fig. 3E; Supplementary Information Section 1.21). The birefringence data are consistent with the box weave 
strand arrangement proposed for 4 . In contrast no birefringence was observed for the unwoven linear polymer 3. Consequences of the long-range order of $\mathbf{4}$ were apparent in in situ deformation caused by applying strain to flakes deposited on a flexible polyester support. The cracking that occurs along clear boundary lines (Fig. 3C) and the slipping between layers (Fig. 3D) are indicative of a material with longrange order and is reminiscent of the response of macroscopic woven textiles to mechanical stress (Supplementary Video 3).

Small-angle X-ray scattering (SAXS) analysis of the metal-coordinated material $4 \cdot[\mathrm{Fe}]$ also shows longrange ordering, with a $d$-spacing consistent with the size of the parent grid (Fig. S32). The wide-angle X-ray scattering (WAXS) of powders of $\mathbf{3}$ and $\mathbf{4}$ are similar (Fig. S33), showing two broad peaks, one at around $2 \theta \sim 25^{\circ}$ (typically associated with aliphatic-aliphatic chain spacing) and the other at $2 \theta \sim 19^{\circ}$ (aromatic-aromatic chain spacing). Such like-with-like phase separation of flexible and rigid segments is typical of block copolymers, and the observation that it occurs for $\mathbf{4}$ suggests that the registry of the aligned strands in the weave is generally maintained in the demetallated 2D molecularly woven material. If strand registry was not conserved a single, very broad, peak would be expected. The broadness of the two peaks corresponding to aliphatic-aliphatic and aromatic-aromatic chain spacings (the $2 \theta$ values indicate these to be on average $\sim 0.36 \mathrm{~nm}$ and $\sim 0.48 \mathrm{~nm}$, respectively) confirm that, despite registry being maintained, every monomer unit does not have exactly the same conformation and intermolecular interactions along the strand. This is unsurprising for a material that is relatively loosely woven from flexible chains aligned through general van der Waals and aromatic stacking interactions. WAXS patterns consisting of broad overlapping peaks showing a similar degree of short-range order have been reported ${ }^{44}$ for poly(amide-block-aramid) alternating block copolymers built from similarly short aliphatic and aromatic units (Fig. S34). The flakes of $\mathbf{4}$ were also imaged using transmission electron microscopy (Figs. S30 \& S31). The electron diffraction patterns consist of streaked rings with diameters corresponding to $d$-spacings in the range $0.38-0.48 \mathrm{~nm}$, showing the same degree of short-range ordering of the aliphatic and aromatic units as the WAXS analysis.

Taken together the characterisation data confirms the layered woven architecture of 4 :

(i) The strands in the model grid [ $\left.\mathrm{Fe}_{9} \mathbf{1}_{6}\right]\left(\mathrm{BF}_{4}\right)_{18}$ were established as woven by X-ray crystallography (Fig. 1). The strands in the tile used for tessellation, $\left[\mathrm{Fe}_{9} \mathbf{2}_{6}\right]\left(\mathrm{BF}_{4}\right)_{18}$, were confirmed as woven from the similarity of the characterisation data (NMR, mass spectrometry etc) of $\left[\mathrm{Fe}_{9} \mathbf{1}_{6}\right]\left(\mathrm{BF}_{4}\right)_{18}$ and $\left[\mathrm{Fe}_{9} \mathbf{2}_{6}\right]\left(\mathrm{BF}_{4}\right)_{18}$. (ii) IR and Raman spectroscopy confirm that polymerisation of the thiols is complete in both $4 \cdot[\mathrm{Fe}]$ and 4 (Figs S17-19).

(iii) AFM and SEM shows that polymerisation of the tiles occurs solely in two-dimensions, not three, with tessellation over large domains leaving no obvious grid-sized gaps within a sheet (although any small gaps in $\mathbf{4} \cdot[\mathrm{Fe}]$ might be filled in $\mathbf{4}$ through strand movement following demetallation; Supplementary Information Section 1.19). Although polymers consisting of alternating short aromaticaliphatic segments do not generally form flake-like morphologies, AFM shows that the 4-nm-thick sheets of 4 are atomically flat for thousands of grid-lengths in two dimensions (Fig. 4C \& D; Fig. S25B). SEM images show displaced sheets of 2D molecularly woven fabric $\mathbf{4}$ that have slid away from others they were apparently stacked with during the formation of 4.[Fe] (Fig. $3 \mathrm{C} \& \mathrm{D}$ ), further confirmation that the sheets are not crosslinked in three dimensions.

(iv) EDS confirms 4 is demetallated.

(v) The strands were aligned and fixed in place by metal coordination in the grid prior to polymerisation. As polymerisation does not remove the metal ions (EDS confirms their presence in 4.[Fe]), the strands must still be aligned, as part of the grid, after within-layer polymerisation.

(vi) After demetallation, when the strands are no longer bound together by metal ions and do have some freedom of movement, birefringence shows the polymer chains are still aligned. The constraints of topology (it is physically impossible for the strands to pass through each other) mean that the aligned polymer chains must still be woven in $\mathbf{4}$, with every one of the nine strand crossings that originate from each grid still present in the material (other than some possible fraying at the edges).

(vii) The like-with-like phase separation revealed by WAXS demonstrates that the registry of the weave is largely maintained in 4: aromatic chain segments associate with aromatic segments of other chains, aliphatic with aliphatic, with the chain spacings averaging $\sim 0.36 \mathrm{~nm}$ and $\sim 0.48 \mathrm{~nm}$, respectively.

(viii) The long-range order (woven architecture) is supported by a wealth of other experimental data, including mechanical fracturing under stress at $90^{\circ}$ angles in the manner of a macroscopic box-weave textile and delamination under stress, further confirmation of the layered structure of the weave. 


\section{Property Comparisons of 3 and 4}

To assess the effects of molecular weaving, we compared the properties of linear 1D polymer 3 and the 2D woven polymer, 4 , using a number of experimental techniques. Preliminary thermogravimetric analysis (TGA) and differential scanning calorimetry (DSC) studies in both air and under an inert atmosphere showed a significant difference in the thermal stability of the materials. TGA demonstrated that the unwoven linear polymer 3 was more stable than the 2D woven polymer $\mathbf{4}$, which showed distinct exotherms at $455^{\circ} \mathrm{C}$ and $490^{\circ} \mathrm{C}$ under air (Fig. S21) that we tentatively assign to the oxidation of the aliphatic and aromatic regions, respectively. It appears that the flake-like morphology of 4 , with a much larger surface-to-volume ratio, facilitates more rapid oxidation.

Young's modulus determination was carried out by AFM using the force-displacement curves obtained at small indentation depths (Fig. S28; Supplementary Video 4). The modulus of $\mathbf{3}$ and $\mathbf{4}$ were measured as $\sim 4.5$ and $\sim 7 \mathrm{GPa}$, respectively, a significant difference in stiffness (by way of comparison, typical values ${ }^{43}$ for nylon, polycarbonate and poly(ethylene terephthalate) (PET) are in the range 2-4 GPa). As would be expected for a woven material, this is consistent with the weaving of the strands in $\mathbf{4}$ holding them more securely in place than would be the case for a more random, spaghetti-like, arrangement of chains, likely present in $\mathbf{3}$.

We also probed the ability of molecular fabric 4 to act as a net, the gaps between the woven warp and weft strands allowing small molecules or ions to pass through but trapping larger ones (Fig. 5; Supplementary Video 5). Syringe-pump filtration of a methanolic suspension of $\mathbf{3}$ or $\mathbf{4}$ through polyvinylidene difluoride (PVDF) with $100 \mathrm{~nm}$ pores resulted in PVDF-supported membranes of $\mathbf{3}$ and 4. SEM analysis of the two membranes showed uniform layers of comparable layer thicknesses, without gaps or deformities (Fig. S36). The membranes were used in ion permeability experiments ${ }^{45}$ that determined the rate of ion transfer through each membrane from high-to-low concentration solutions using a small $\mathrm{Fe}(\mathrm{II})$ (phen) ${ }_{3}$ complex (diameter $=1.3 \mathrm{~nm}$ ) and a larger $\mathrm{Fe}(\mathrm{II})$-circular helicate (diameter $=2.5 \mathrm{~nm}$ ) (Fig. 5A \& 5B). The PVDF-supported membranes of $\mathbf{3}$ and $\mathbf{4}$ allowed permeation of the smaller $\mathrm{Fe}(\mathrm{II})$ (phen) ${ }_{3}$ complex at similar transfer rates (approximately $2 \%$ of the feed concentration over 5 hours) and solute rejection values (52-58\%, Fig. 5D). However, the membranes had significantly different rates of transfer for the larger $\mathrm{Fe}(\mathrm{II})$-circular-helicate $(6-10 \times$ faster with 3; Fig. $5 \mathrm{C})$. The loose tangle of polymer molecules in $\mathbf{3}$ allows the passage of the large $\mathrm{Fe}$ (II)-circular-helicate cation (solute rejection value $6 \%$, Fig. 5D) while the woven geometry of 4 restricts the number of gaps that are of sufficient size for this larger cation to pass through (solute rejection value 90\%, Fig. 5D). Further permeability studies on charged and uncharged species of different shapes and sizes are ongoing. 

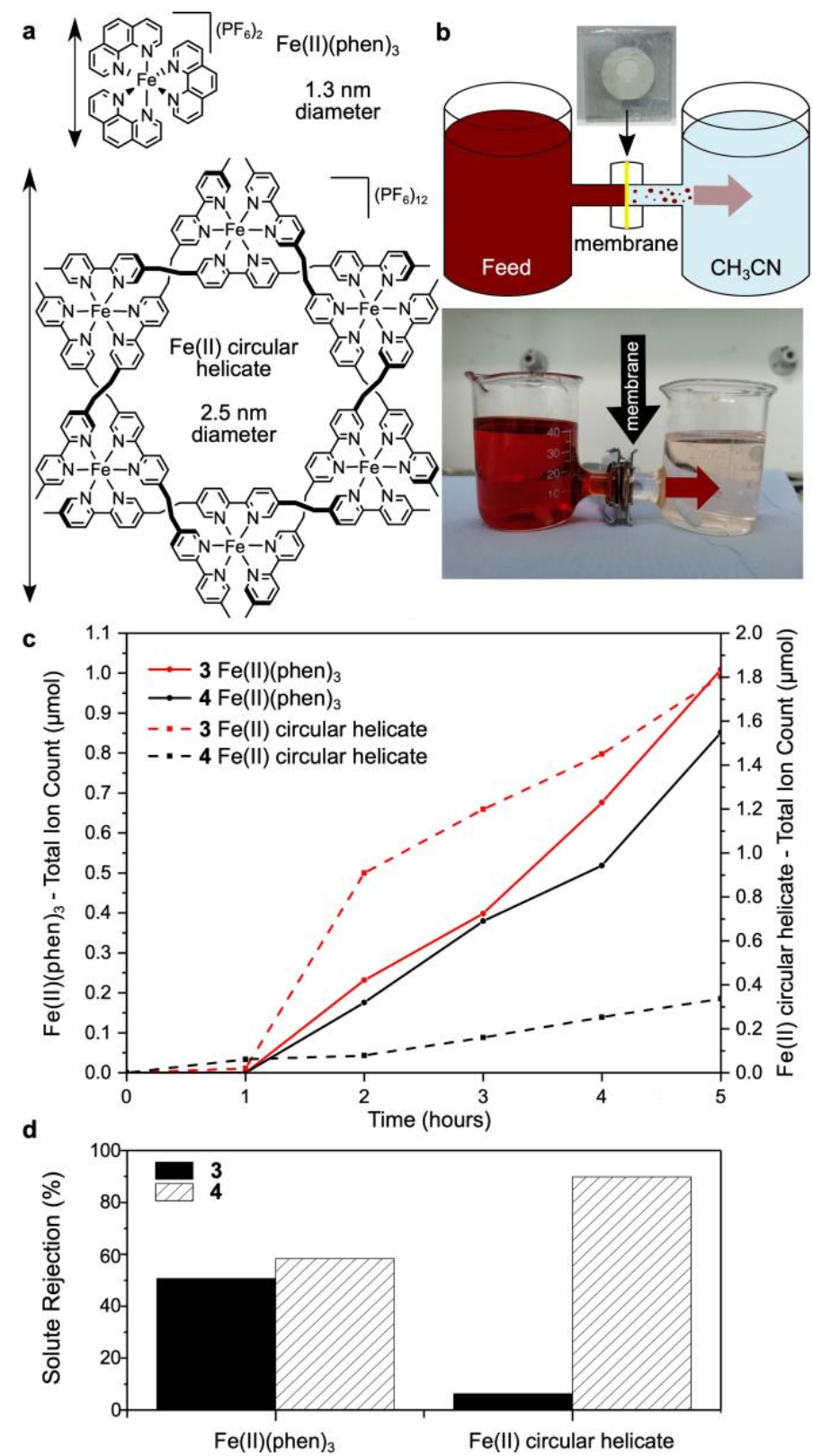

Figure 5. Ion permeability studies on PVDF-supported membranes formed from unwoven linear polymer 3 and 2D woven polymer 4. (A) Structures of the Fe(II) complexes used. (B) Experimental setup for the ion permeation experiments. (C) Plot comparing the diffusion rates across PVDFsupported membranes of $\mathbf{3}$ and $\mathbf{4}$ for $\mathrm{Fe}$ (II) complexes of different sizes. (D) Plot of the relative solute rejection for the different ions across PVDF-supported membranes of $\mathbf{3}$ and $\mathbf{4}$ at $5 \mathrm{~h}$. For an animation of the ion permeability studies, see Supplementary Video 5.

\section{Discussion}

As the difference between stacked ${ }^{36,37}$ and interwoven grids illustrates, the regular repetition of a motif where two strands simply cross each other is, not in itself, sufficient to generate a mechanically entangled structure. 3D woven COFs have previously been formed ${ }^{12}$ from tetrahedral building blocks of bidentate ligands with a single crossing, relying on reticular assembly to generate the entanglements necessary for a weave. However, reticular considerations are insufficient to ensure that a framework of coordination tetrahedra that feature a single crossing generate a lattice that is woven: a weave only results if all adjacent tetrahedra are internally oriented so that the correct ligand ends are connected. Likewise, a recently reported ${ }^{46}$ ring-opening polymerisation of a [2]catenane cannot control the crossing sequence nor the orientation of warp and weft strands to ensure that the resulting polymer chains are woven rather than randomly entangled. This contrasts with the approach used to assemble molecularly 
woven fabric 4, in which building blocks that are pre-woven are tessellated to extend an already established weave. The resulting demetallated material consists of wholly organic polymer chains with orthogonal warp and weft strands woven in two-dimensional sheets of uniform $4 \mathrm{~nm}$ thickness, although the synthetic strategy means some imperfections in the weave will inevitably occur from chains being incorrectly connected (Supplementary Information Section 1.19). Following the demetallation, AFM and SEM show individual sheets of the 2D molecularly woven fabric 4 (e.g. Fig. S25B) demonstrating that the weave is restricted to two dimensions and that the sheets are not crosslinked vertically. Molecularly woven material $\mathbf{4}$ is formally a $2 \mathrm{D}$ woven covalent organic framework (2D-woven-COF), but it is not made of the short rigid repeat units typical of COFs that promote crystallinity and $\mathbf{4}$ has the modest short-range order typical of aligned loosely packed polymer chains (i.e. every repeat unit does not simultaneously adopt exactly the same conformation and intermolecular interactions). It may be easier for materials that are not crystalline, nor derived wholly from rigid constituents, to realise properties that can be imparted by the long-range order of weaving.

Advances in the 'top-down' weaving of one-dimensional strands-ranging from threads with diameters measured in millimetres (reeds, plant fibres, etc) to those of a few microns (wool, cotton, synthetic polymers, etc)-in two-dimensions have underpinned technological progress through the ages. ${ }^{1}$ As with macroscopic yarns, the weaving of flexible one-dimensional molecular strands into a layered fabric results in a woven material with an array of characteristics and properties that are significantly different to those of the unwoven fibers. The ability to weave polymer chains in two (and potentially three) dimensions by the tessellation and reticulation of pre-woven molecular building blocks offers new opportunities and research directions that combine the fields of polymer structure and topology, ${ }^{47}$ twodimensional materials ${ }^{17,18,48}$ and the molecular-level mechanical bond. ${ }^{49,50}$

\section{Methods}

\section{Synthesis of $3 \times 3$ interwoven grid $\left[\mathrm{Fe}_{9} 2_{6}\right]\left(\mathrm{BF}_{4}\right)_{18}$}

Ligand $2(49.7 \mathrm{mg}, 43.1 \mu \mathrm{mol})$ and $\mathrm{Fe}\left(\mathrm{BF}_{4}\right)_{2} \cdot 6 \mathrm{H}_{2} \mathrm{O}(21.8 \mathrm{mg}, 1.5 \mathrm{eq} ., 64.6 \mu \mathrm{mol})$ were suspended in acetonitrile $(6.2 \mathrm{~mL})$ and toluene $(3.7 \mathrm{~mL})$ in a microwave vessel under $\mathrm{N}_{2}$ atmosphere. The mixture was heated at $120^{\circ} \mathrm{C}$ in a microwave for $20 \mathrm{~h}$. After cooling, the mixture was diluted with diethyl ether and the resulting dark green precipitate filtered onto celite, washed with diethyl ether and redissolved in acetonitrile. After solvent evaporation, the resulting dark green powder was kept under nitrogen (46 $\mathrm{mg}, 4.5 \mu \mathrm{mol}, 62 \%$ yield) or alternatively dissolved in acetonitrile for immediate use. For characterisation data see Supplementary Information.

\section{Tessellation and Polymerisation of $\left[\mathrm{Fe}_{9} 2_{6}\right]\left(\mathrm{BF}_{4}\right)_{18}$ to form layered metal-coordinated 2D molecularly woven fabric $4 \cdot[\mathrm{Fe}]$}

Freshly prepared [Feg26](BF4) 18 (12.2 mg, $1.2 \mu \mathrm{mol}$ based on $100 \%$ conversion from ligand 2 ) was dissolved in a mixture of toluene $(1 \mathrm{~mL})$ and acetonitrile $(1.67 \mathrm{~mL})$. After slow precipitation and concomitant polymerisation in air over 3 days, a green film and microcrystalline particles had collected on the surfaces of the reaction vessel. This material was washed repeatedly with acetonitrile to give the product as a dark/green solid $(8.0 \mathrm{mg}, 66 \%)$. For characterisation data see Supplementary Information.

\section{De-metallation of 4.[Fe] to form wholly-organic 2D molecularly woven fabric 4}

Material 4. $[\mathrm{Fe}](8.0 \mathrm{mg})$ was suspended in $3 \mathrm{~mL}$ DMSO with $15.0 \mathrm{mg}$ of KCN (300 eq., approx. 5 times the amount required to fully complex all of the $\mathrm{Fe}$ (II) cations). The dark green turbid solution was left at room temperature for 16 hours. The resulting turbid dark orange solution was diluted to $5 \mathrm{~mL}$ with DMSO and centrifuged. The supernatant was discarded; the orange solid was suspended in $5 \mathrm{~mL}$ of DMSO and centrifuged again. The process was repeated until the DMSO was colourless (typically four times) and then twice with ethanol and other solvents. The solid was dried under vacuum and the material was isolated as a pale yellow-orange powder $4(4.6 \mathrm{mg}, 72 \%$ yield). For characterisation data see Supplementary Information.

\section{References}

1. Kadolph, S. J. ed. Textiles (Saddle River, New Jersey: Prentice-Hall, ed. 10, 2007).

2. Batten, S. R. \& Robson, R. Interpenetrating nets: ordered, periodic entanglement. Angew. Chem. Int. Ed. 37, 1460-1494 (1998).

3. Carlucci, L., Ciani, G. \& Proserpio, D. M. Polycatenation, polythreading and polyknotting in coordination network chemistry. Coord. Chem. Rev. 246, 247-289 (2003). 
4. Van Calcar, P. M., Olmstead, M. M. \& Balch, A. L. Construction of a knitted crystalline polymer through the use of Gold(I)-Gold(I) interactions. Chem. Commun. 17, 1773-1774 (1995).

5. Axtell III, E. A., Liao, J.-H. \& Kanatzidis, M. G. Flux synthesis of LiAuS and NaAuS: "Chicken-wirelike" layer formation by interweaving of (AuS) $n^{\text {n- }}$ threads. Comparison with $\alpha-H g S$ and $A A u S(A=$ K, Rb). Inorg. Chem. 37, 5583-5587 (1998).

6. Carlucci, L., Ciani, G., Gramaccioli, A., Proserpio, D. M. \& Rizzato, S. Crystal engineering of coordination polymers and architectures using the $\left[\mathrm{Cu}\left(2,2^{\prime}-\text { bipy }\right)\right]^{2+}$ molecular corner as building block (bipy = 2,2'- bipyridyl). Cryst. Eng. Commun. 29, 1-10 (2000).

7. Li, Y.-H., Su, C.-Y., Goforth, A. M., Shimizu, K. D., Gray, K. D., Smith, M. D. \& zur Loye, H.-C. The first 'two-over/two-under' (2O/2U) 2D weave structure assembled from Hg-containing 1D coordination polymer chains. Chem. Commun. 14, 1630-1631 (2003).

8. Han, L. \& Zhou, Y. 2D Entanglement of 1D flexible zigzag coordination polymers leading to an interwoven network. Inorg. Chem. Commun. 11, 385-387 (2008).

9. Wu, H., Yang, J., Su, Z.-M., Batten, S. R. \& Ma, J.-F. An exceptional 54-fold interpenetrated coordination polymer with $10^{3}$-srs network topology. J. Am. Chem. Soc. 133, 11406-11409 (2011).

10. Champsaur, A. M., Méziére, C., Allain, M., Paley, D. W., Steigerwald, M. L., Nuckolls, C. \& Batail, P. Weaving nanoscale cloth through electrostatic templating. J. Am. Chem. Soc. 139, 1171811721 (2017).

11. Ciengshin, T., Sha, R. \& Seeman, N. C. Automatic molecular weaving prototyped by using singlestranded DNA. Angew. Chem. Int. Ed. 50, 4419-4422 (2011).

12. Liu, Y., Ma, Y., Zhao, Y., Sun, X., Gándara, F., Furukawa, H., Liu, Z., Zhu, H., Zhu, C., Suenaga, K., Oleynikov, P., Alshammari, A. S., Zhang, X., Terasaki, O. \& Yaghi, O. M. Weaving of organic threads into a crystalline covalent organic framework. Science 351, 365-369 (2016).

13. Zhao, Y., Guo, L., Gándara, F., Ma, Y., Liu, Z., Zhu, C., Lyu, H., Trickett, C. A., Kapustin, E. A., Terasaki, O. \& Yaghi, O. M. A synthetic route for crystals of woven structures, uniform nanocrystals, and thin films of imine covalent organic frameworks. J. Am. Chem. Soc. 139, 13166-13172 (2017).

14. Liu, Y., Ma, Y., Yang, J., Diercks, C. S., Tamura, N., Jin, F. \& Yaghi, O. M. Molecular weaving of covalent organic frameworks for adaptive guest inclusion. J. Am. Chem. Soc. 140, 16015-16019 (2018).

15. Lewandowska, U., Zajaczkowski, W., Corra, S., Tanabe, J., Borrmann, R., Benetti, E. M., Stappert, S., Watanabe, K., Ochs, N. A. K., Schaeublin, R., Li, C., Yashima, E., Pisula, W., Müllen, K. \& Wennemers, H. A triaxial supramolecular weave. Nat. Chem. 9, 1068-1072 (2017).

16. Wang, Z., Błaszczyk, A., Fuhr, O., Heissler, S., Wöll, C. \& Mayor, M. Molecular weaving via surface-templated epitaxy of crystalline coordination networks. Nat. Commun. 8, 14442 (2017).

17. Servalli, M. \& Schlüter, A. D. Synthetic two-dimensional polymers. Annu. Rev. Mater. Res. 47, 361-389 (2017).

18. Mas-Ballesté, R., Gómez-Navarro, C., Gómez-Herrero, J. \& Zamora, F. 2D materials: to graphene and beyond. Nanoscale 3, 20-30 (2011).

19. Busch, D. H. Structural definition of chemical templates and the prediction of new and unusual materials. J. Inclusion Phenom. Mol. Recognit. Chem. 12, 389-395 (1992).

20. Hubin, T. J. \& Busch, D. H. Template routes to interlocked molecular structures and orderly molecular entanglements. Coord. Chem. Rev. 200-202, 5-52 (2000).

21. Cockriel, D. L., McClain, J. M., Patel, K. C., Ullom, R., Hasley, T. R., Archibald, S. J. \& Hubin, T. $\mathrm{J}$. The design and synthesis of pyrazine amide ligands suitable for the "tiles" approach to molecular weaving with octahedral metal ions. Inorg. Chem. Commun. 11, 1-4 (2008).

22. Wadhwa, N. R., Hughes, N. C., Hachem, J. A. \& Mezei, G. Metal-templated synthesis of intertwined, functionalized strands as precursors to molecularly woven materials. RSC Adv. 6, $11430-11440(2016)$.

23. Mena-Hernando, S. \& Pérez, E. M. Mechanically interlocked materials. Rotaxanes and catenanes beyond the small molecule. Chem. Soc. Rev. 48, 5016-5032 (2019).

24. Forgan, R. S., Sauvage, J.-P. \& Stoddart, J. F. Chemical topology: complex molecular knots, links, and entanglements. Chem. Rev. 111, 5434-5464 (2011).

25. Ayme, J.-F., Beves, J. E., Campbell, C. J. \& Leigh, D. A. Template synthesis of molecular knots. Chem. Soc. Rev. 42, 1700-1712 (2013).

26. Sauvage, J.-P. From chemical topology to molecular machines (Nobel Lecture), Angew. Chem. Int. Ed. 56, 11080-11093 (2017).

27. Dietrich-Buchecker, C. O. \& Sauvage, J.-P. A synthetic molecular trefoil knot. Angew. Chem. Int. Ed. Engl. 28, 189-192 (1989). 
28. Chichak, K. S., Cantrill, S. J., Pease, A. R., Chiu, S.-H., Cave, G. W. V., Atwood, J. L. \& Stoddart, J. F. Molecular Borromean rings. Science 304, 1308-1312 (2004).

29. Guo, J., Mayers, P. C., Breault, G. A. \& Hunter, C. A. Synthesis of a molecular trefoil knot by folding and closing on an octahedral coordination template. Nat. Chem. 2, 218-222 (2010).

30. Ayme, J.-F., Beves, J. E., Leigh, D. A., McBurney, R. T., Rissanen, K. \& Schultz, D. A synthetic molecular pentafoil knot. Nat. Chem. 4, 15-20 (2012).

31. Prakasam, T., Lusi, M., Elhabiri, M., Platas-Iglesias, C., Olsen, J.-C., Asfari, Z., CianféraniSanglier, S., Debaene, F., Charbonnière, L. J. \& Trabolsi, A. Simultaneous self-assembly of a [2]catenane, a trefoil knot, and a Solomon link from a simple pair of ligands. Angew. Chem., Int. Ed. 52, 9956-9960 (2013).

32. Wood, C. S., Ronson, T. K., Belenguer, A. M., Holstein, J. J. \& Nitschke, J. R. Two-stage directed self-assembly of a cyclic [3]catenane. Nat. Chem. 7, 354-358 (2015).

33. Danon, J. J., Krüger, A., Leigh, D. A., Lemonnier, J.-F., Stephens, A. J., Vitorica-Yrezabal, I. J. \& Woltering, S. L. Braiding a molecular knot with eight crossings. Science 355, 159-162 (2017).

34. Zhang, L., Stephens, A. J., Nussbaumer, A. L., Lemonnier, J.-F., Jurček, P., Vitorica-Yrezabal, I. J. \& Leigh, D. A. Stereoselective synthesis of a composite knot with nine crossings. Nat. Chem. 10, 1083-1088 (2018).

35. Leigh, D. A., Schaufelberger, F., Pirvu, L., Halldin Stenlid, J., August, D. P. \& Segard, J. Tying different knots in a molecular strand. Nature 584, 562-568 (2020).

36. Ruben, M., Rojo, J., Romero-Salguero, F. J., Uppadine, L. H. \& Lehn, J.-M. Grid-type metal ion architectures: functional metallosupramolecular arrays. Angew. Chem. Int. Ed. 43, 3644-3662 (2004).

37. Dawe, L. N., Abedin, T. S. M. \& Thompson, L. K. Ligand directed self-assembly of polymetallic [n $\times$ n] grids: rational routes to large functional molecular subunits? Dalton Trans. 1661-1675 (2008).

38. Leigh, D. A., Danon, J. J., Fielden, S. D. P., Lemonnier, J.-F., Whitehead, G. F. S. \& Woltering, S. L. A molecular endless (74) knot. In press.

39. Pakula, A. A. \& Simon, M. I. Determination of transmembrane protein structure by disulfide crosslinking: The Escherichia coli Tar receptor. Proc. Natl. Acad. Sci. USA 89, 4144-4148 (1992).

40. Sakamoto, R., Takada, K., Pal, T., Maeda, H., Kambe, T. \& Nishihara, H. Coordination nanosheets (CONASHs): strategies, structures and functions. Chem. Commun. 53, 5781-5801 (2017).

41. De Gennes, P. G. Reptation of a polymer chain in the presence of fixed obstacles. J. Chem. Phys. 55, 572-571 (1971).

42. Zhu, W., Low, T., Perebeinos, V., Bol, A. A., Zhu, Y., Yan, H., Tersoff, J. \& Avouris, P. Structure and electronic transport in graphene wrinkles. Nano Lett. 12, 3431-3436 (2012).

43. Young, R. J. \& Lovell, P. A. Introduction to polymers (CRC Press, Boca Raton, FL, ed. 3, 2011).

44. de Ruijter, C., Mendes, E., Boerstoel, H. \& Picken, S. J. Orientational order and mechanical properties of poly(amide-block-aramid) alternating block copolymer films and fibers. Polymer 47, 8517-8526 (2006).

45. Kontturi, K., Murtomäki, L. \& Manzanares, J. A. Ionic Transport Processes: in Electrochemistry and Membrane Science (Oxford Univ. Press, 2014).

46. Li, G., Wang, L., Wu, L., Guo, Z., Zhao, J., Liu, Y., Bai, R. \& Yan, X. Woven polymer networks via the topological transformation of a [2]catenane. J. Am. Chem. Soc. 142, 14343-14349 (2020).

47. Hawker, C. J. \& Wooley, K. L. The convergence of synthetic organic and polymer chemistries. Science 309, 1200-1205 (2005).

48. Sakamoto, J., van Heijst, J., Lukin, O. \& Schlüter, A. D. Two-dimensional polymers: just a dream of synthetic chemists? Angew. Chem. Int. Ed. 48, 1030-1069 (2009).

49. Wu, Q., Rauscher, P. M., Lang, X., Wojtecki, R. J., de Pablo, J. J., Hore, M. J. A. \& Rowan, S. J. Poly[n]catenanes: Synthesis of molecular interlocked chains. Science 358, 1434-1439 (2017).

50. Stoddart, J. F.; Dawning of the age of molecular nanotopology. Nano. Lett. 20, 5597-5600 (2020).

\section{Acknowledgements}

We thank the Engineering and Physical Sciences Research Council (EPSRC; EP/P027067/1), the European Research Council (ERC; Advanced Grant no. 786630), and the Defense Advanced Research Projects Agency (DARPA; Co-operative Agreement W911NF-17-2-0148) for funding; with networking contributions from the COST Action CA17139, EUTOPIA. The views, opinions and/or findings expressed are those of the authors and should not be interpreted as representing the official views or policies of the Department of Defense or the U.S. Government. We also thank the Diamond Light Source (UK) for synchrotron beam time on 119 (XR029), the University of Manchester, Department of 
Chemistry microanalysis and mass spectrometry services, the Henry Royce Institute for Advanced Materials (funded through EPSRC grants EP/R00661X/1 and EP/P025021/1) for the use of facilities, Stuart Jantzen/Biocinematics for the video animations, and Profs S. J. Rowan (University of Chicago) and R. P. Sijbesma (Eindhoven University) for comments that improved the draft manuscript. D.A.L. is a Royal Society Research Professor.

\section{Author contributions}

D.P.A., L.I.P., J.F.L. and Y.S. carried out the synthesis and general characterisation studies. G.F.S.W. solved the crystal structure of [Fe $\left.{ }_{9} 1_{6}\right]\left(B F_{4}\right)_{18}$. Z.L., C.A.M. and R.J.Y carried out the AFM studies. Z.L. and R.J.Y. performed the Young's modulus, polarised optical microscope and deformation experiments. S.J.H conducted the TEM studies, and R.A.W.D. and P.R.C.K. the ion permeation studies. D.A.L. directed the research. All authors contributed to the analysis of the results and the writing of the manuscript. Authors are listed alphabetically in view of the broad range of experimental techniques used in this study.

\section{Competing Interests statement}

The authors declare no competing interests.

Additional information: Supplementary Information is available for this paper. Correspondence and requests for materials should be addressed to David A. Leigh.

\section{Data availability}

The data that support the findings of this study are available within the paper and its Supplementary Information, or are available from the Mendeley data repository (https://data.mendeley.com/) under doi XXXXxx. 